\title{
Modeling of Microwave Active Devices Using the FDTD Analysis Based on the Voltage-Source Approach
}

\author{
Chien-Nan Kuo, Ruey-Beei Wu, Bijan Houshmand, and Tatsuo Itoh, Fellow, IEEE
}

\begin{abstract}
This letter describes a voltage-source-based formulation of the extended finite-difference time-domain algorithm for the purpose of modeling microwave devices. The device-wave interaction is fully characterized by replacing the lumped devices with equivalent voltage sources in the device region, which in turn generate electromagnetic fields according to Faraday's law. This formulation is applied to the analysis of a typical microwave amplifier, which includes a three-terminal active MESFET device. Simulation results are in good agreement with measured data.
\end{abstract}

\section{INTRODUCTION}

W ITH the advancement of computers, the finitedifference time-domain (FDTD) method has become a very popular tool for analysis of various electromagnetic problems, including microwave circuits. As the size of microwave circuits and spacing between circuit elements become smaller, the coupling between the closely spaced elements adds more complicated effects to circuit performances. Proper modeling of the lumped passive and/or active devices and electromagnetic waves is critical in circuit simulation. Extensive investigations have been presented in literatures aiming at the extension of the FDTD method to include lumped microwave devices in the full-wave analysis. The FDTD method has been extended to incorporate circuit quantities of a two-terminal device into the algorithm [1]-[3]. By recognizing that the equivalent circuit model consists of two-terminal lumped elements, three-terminal active devices can be modeled by incorporating these elements into several FDTD cells [4], [5]. This approach, however, may become quite cumbersome in the modeling of active devices with complicated equivalent circuit models. A more general approach substitutes the device with equivalent current sources and consequently allows direct access to all SPICE models in the FDTD calculation [6]. This approach has been found to be advantageous in the simulation of practical three-dimensional circuits such as amplifiers [7], [8].

Manuscript received November 16, 1995. This work was supported by Hughes Micro, JSEP contract F49620-92-C-0055, and Army Research Office contract DAAH04-93-G-0068.

C.-N. Kuo and T. Itoh are with the Electrical Engineering Department, University of California at Los Angeles, CA 90095 USA.

R.-B. Wu is with the Electrical Engineering Department, National Taiwan University, Taipei, Taiwan.

B. Houshmand is with the Jet Propulsion Laboratory, Pasadena, CA 91109 USA.

Publisher Item Identifier S 1051-8207(96)03431-9.
In the previous approach, the lumped devices are treated as current sources that interact with electromagnetic fields in accordance with Ampere's law. This letter presents a dual approach that employs voltage sources to represent the lumped devices and generate electromagnetic fields according to Faraday's law. The formulation is established to construct an equivalent circuit of the FDTD meshes as seen from the device. The approach is then applied to simulate practical three-dimensional microstrip amplifier circuits.

\section{FORMULATION}

An active device in a microwave circuit is typically very small in size compared to a wavelength, and it can be modeled by its equivalent lumped circuit with a very high degree of accuracy. In order to include the equivalent circuit model into the FDTD analysis, the active device can be replaced by equivalent voltage sources in the active region if the voltage sources satisfy the voltage-current relationship at the input/output ports and the scattering properties of the active device.

Consider an active device in a microstrip circuit. Fig. 1 shows the placement of the voltage sources at one port of the active device; each source is aligned to the FDTD grid edges beneath and perpendicular to the microstrip line with each ends connected to the microstrip line or a grounded via. Those vias, modeled as perfect conductors, provide a voltage reference to the voltage sources. Assume that the voltage sources lie in an active sheet parallel to the $x-y$ plane. Without loss of generality, consider a voltage source with its center denoted by $\left(i, j+\frac{1}{2}, k\right)$, where two FDTD meshes contribute to the current flowing through the voltage source. For the right mesh, the integral form of Faraday's law can be expressed in terms of circuit quantities as

$$
-L_{1} \frac{d I_{\mathrm{mesh}, 1}}{d t}=V_{\mathrm{loop}, 1}+V_{\mathrm{dev}}
$$

where $L_{1}$ is the space inductance of FDTD cells, $V_{\text {loop, } 1}$ is the equivalent voltage obtained from the the loop integration of the $E$-field along the cell boundary other than the edge of the voltage source, and $I_{\text {mesh,1 }}$ is the loop current that flows along the right-hand side of the active sheet through the cell width $\Delta x$. A similar formulation can be derived for the left mesh to obtain a circuit equation for the loop current $I_{\text {mesh,2 }}$ which flows along the left-hand side of the active sheet. The device current $I_{\mathrm{dev}}$ flowing into the voltage source equals the 


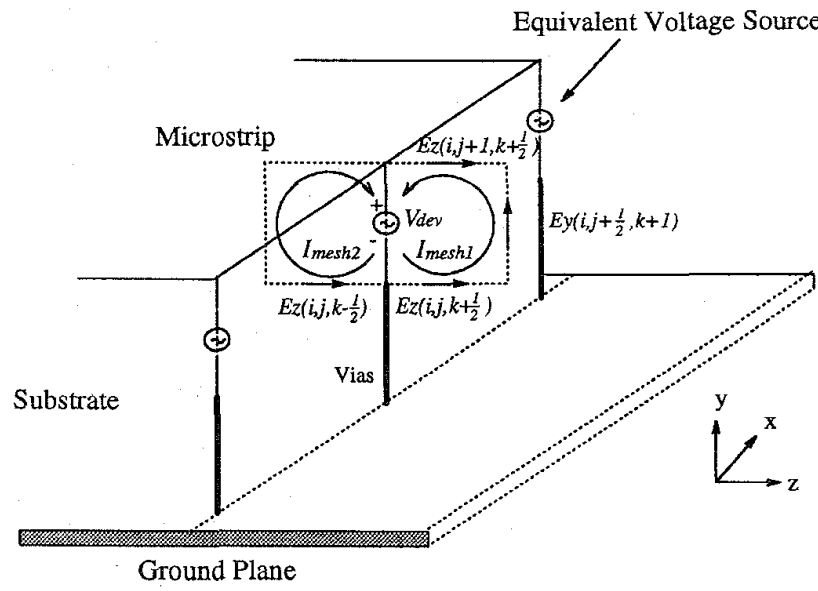

Fig. 1. The replacement of the active device by equivalent voltage sources on the FDTD grid edges.

sum of the two loop current components on both sides of the active sheet.

In the case that there are $N$ voltage sources placed across the entire width of the microstrip line, the device current $I_{\mathrm{dev}}$ is the sum of all the loop currents. The governing equation is derived as

$$
-L_{\text {total }} \frac{d I_{\mathrm{dev}}}{d t}=V_{\mathrm{total}}+V_{\mathrm{dev}}
$$

where

$$
\begin{aligned}
V_{\text {total }} & =\sum_{i}^{2 N} \frac{V_{\text {loop }, i}}{L_{i} / L_{\text {total }}} \\
L_{\text {total }} & =1 / \sum_{i}^{2 N} \frac{1}{L_{i}}
\end{aligned}
$$

and $L_{i}$ is the space inductance of each mesh. The equivalent circuit of the governing equation is shown in Fig. 2. The voltage source/inductor pair is the equivalent circuit as seen from the device. Through this equivalent circuit, the field quantities in the FDTD algorithm are combined with the circuit quantities in the device model to describe the interaction between the device and electromagnetic fields.

The extended FDTD time-marching scheme can be described as follows:

Step 0) The electric fields $E^{n-1}$, magnetic fields $H^{n-\frac{1}{2}}$, device voltage $V_{\mathrm{dev}}^{n-\frac{1}{2}}$, and device current $I_{\mathrm{dev}}^{n-\frac{1}{2}}$ are given, where the superscript denotes the time step.

Step 1) The update of electric fields $E^{n}$ is formed from $E^{n-1}$ and $H^{n-\frac{1}{2}}$ by employing Ampere's law. Note that the electric fields on the active sheet have yet to be determined since Ampere's law is not applicable in the device region.

Step 2) The voltage source $V_{\text {total }}^{n}$ is calculated from nearby electric fields by (3). The FDTD meshes as seen from the device satisfy (2). Combining (2) and the device circuit model, the values of the device voltage and current at the next time step, $V_{\mathrm{dev}}^{n+\frac{1}{2}}$ and $I_{\mathrm{dev}}^{n+\frac{1}{2}}$, can be solved by using typical circuit

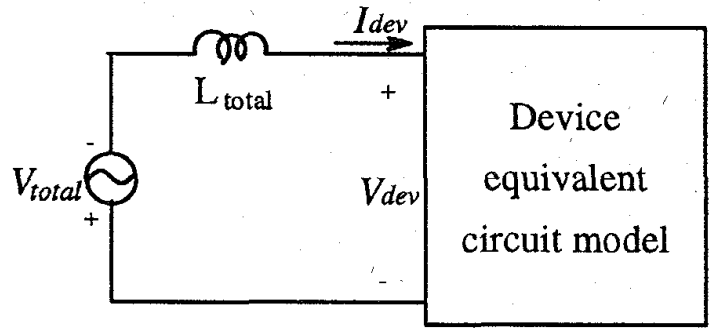

Fig. 2. The equivalent circuit of the configuration in Fig. 1, as seen from the device.

approaches, e.g., the backward difference scheme for the state equation [9], with the initial values of $V_{\mathrm{dev}}^{n-\frac{1}{2}}$ and $I_{\mathrm{dev}}^{n-\frac{1}{2}}$, respectively. The electric fields $E^{n}$ on the active sheet are then determined by the device voltage $V_{\mathrm{dev}}^{n}$, which is approximated by the average of $V_{\mathrm{dev}}^{n+\frac{1}{2}}$ and $V_{\mathrm{dev}}^{n-\frac{1}{2}}$

Step 3) The magnetic fields $H^{n+\frac{1}{2}}$ are updated from $H^{n-\frac{1}{2}}$ and $E^{n}$ by employing Faraday's law.

It is worth noting that Steps 1) and 3) in the updating algorithm for the electric and magnetic fields are exactly the same as those in the conventional FDTD algorithm.

\section{APPLICATIONS}

The model is applied to analyze an actual microwave amplifier as in [8], which includes dc biasing circuits, impedance-matching circuits, and a three-terminal GaAs MESFET, NE72084. The layout of the amplifier and the equivalent lumped circuit model of the active device are depicted in Figs. 1 and 2(b) of [8].

The FDTD simulation is performed with uniform grids of space steps $\Delta x=13 \mathrm{mil}, \Delta y=7.5 \mathrm{mil}$, and $\Delta z=10$ mil. The entire computation domain is divided into a grid of 90 (width) $\times 25$ (height) $\times 230$ (length) cells. Higdon's secondorder absorbing boundary condition is used as in [10]. The radial stubs in the dc biasing circuits is modeled by the staircase approximation. The lumped resistors and capacitors in biasing circuits and matching circuits are treated as distributed elements and incorporated into the FDTD algorithm as in [1] The dc power supply is modeled as an RF short circuit. The active region occupies eight cells in the longitudinal direction for the modeling of the packaged active device. For this twoport device, two sets of voltage sources are placed at the gate and drain ports. The numbers of voltage sources at the gate and drain ports are eight and four, respectively.

The $S$-parameters of the amplifier are calculated by taking the Fourier transform of the observed time response. Calculations show that the gain at $6 \mathrm{GHz}$ is $9.33 \mathrm{~dB}$ and the matching point of the $\left|S_{11}\right|$ curve is $5.75 \mathrm{GHz}$. Measured data and the results of using current-source approach as in [8] are plotted in Fig. 3 for comparison. In Fig. 3(a), measured data smaller than $-2 \mathrm{~dB}$ are truncated because of the choice of the scale in experiment. The matching point of measured data is at 5.6 $\mathrm{GHz}$, and the gain is $9.23 \mathrm{~dB}$ at $6 \mathrm{GHz}$. The results of the voltage-source approach and the current-source approach are very similar, since both approaches are dual of each other. The 


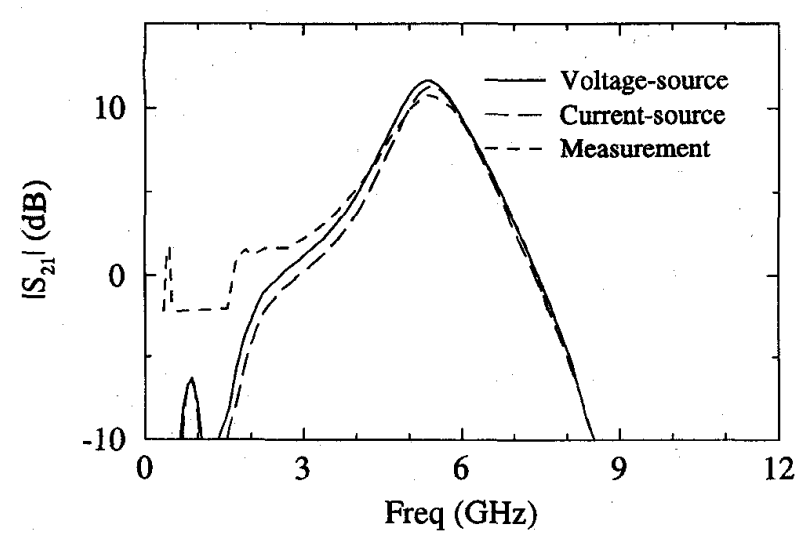

(a)

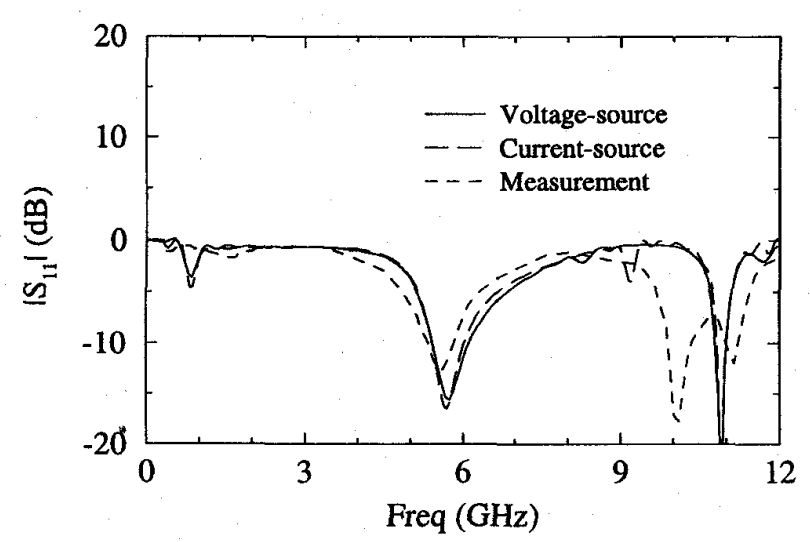

(b)

Fig. 3. The calculated results of two different approaches and measured $S$-parameters. (a) $\left|S_{21}\right|$. (b) $\left|S_{11}\right|$.

matching point is shifted if a different number of equivalet voltage sources is employed. The trend is the same as that in [11], which uses equivalent current sources. The spike in the $\left|S_{21}\right|$ curve at about $1 \mathrm{GHz}$ is related to low-frequency oscillation. It has been found that the modeling of the dc power supply strongly affects the magnitude of the spike. In any case, FDTD simulations can predict those out-of-band dips near 1 and $11 \mathrm{GHz}$ in the $\left|S_{11}\right|$ curve.

\section{CONCLUSION}

A voltage-source approach is proposed for the extended FDTD analysis of microwave circuits, which may include three-terminal active devices. In this approach, the active device, substituted by equivalent voltage sources in the active region, can be viewed as a black box. The approach is then applied to the analysis of an actual microwave amplifier circuit. Simulation results show good agreement with measured data. In general, the voltage-source approach can be used for multi-port active devices. Utilizing equivalent voltage sources to model the device-wave interaction, the extended FDTD method can analyze the entire microwave circuit as a whole.

\section{REFERENCES}

[1] W. Sui, D. A. Christensen, and C. H. Durney, "Extending the twodimensional FD-TD method to hybrid electromagnetic systems with active and passive lumped elements," IEEE Trans. Microwave Theory Tech., vol. 40, pp. 724-730, Apr. 1992.

[2] B. Toland, B. Houshmand, and T. Itoh, "Modeling of nonlinear active regions with the FDTD method," IEEE Microwave Guided Wave Lett., vol. 3, no. 9, pp. 333-335, Sept. 1993.

[3] B. Toland, J. Lin, B. Houshmand, and T. Itoh, "FDTD analysis of an active antenna," IEEE Microwave Guided Wave Lett., vol. 3, no. 11, pp. 423-425, Nov. 1993.

[4] Y.-S. Tsuei, A. C. Cangellaris, and J. L. Prince, "Rigorous electromagnetic modeling of chip-to-package (first-level) internonnections," IEEE Trans. Components, Hybrids, and Manufacturing Tech., vol. 16, pp. $876-883$, Dec. 1993

[5] M. Piket-May, A. Taflove, and J. Baron, "FD-TD modeling of digital signal propagation in 3-D circuits with passive and active loads," IEEE Trans. Microwave Theory Tech., vol. 42, no. 8, pp. 1514-1523, Aug. 1994.

[6] V. A. Thomas, M. E. Jones, M. Piket-May, A. Taflove, and E. Harrigan, "The use of SPICE lumped circuits as subb-grid models for FDTD analysis," IEEE Microwave Guided Wave Lett., vol. 4, no. 5, pp. 141-143, May 1994.

[7] C.-N. Kuo, S. T. Chew, B. Houshmand, and T. Itoh, "FDTD simulation of a microwave amplifier," in IEEE MTT-S Int. Microwave Symp., Orlando, FL, May 1995.

[8] C.-N. Kuo, V. A. Thomas, S. T. Chew, B. Houshmand, and T. Itoh, "Small signal analysis of active circuits using FDTD algorithm," IEEE Microwave Guided Wave Lett., vol. 5, no. 7, pp. 216-218, July 1995.

[9] C. A. Desoer and E. S. Kuh, Basic Circuit Theory. New York: McGraw-Hill, 1969, chs. 12, 16.

[10] R. L. Higdon, "Absorbing boundary conditions for difference approximations to the multi-dimensional wave equation," Math. Comput., vol. 47, no. 176 , pp. $437-459$, Oct. 1986.

[11] C.-N. Kuo, B. Houshmand, and T. Itoh, "FDTD analysis of active circuits with equivalent current source approach," in IEEE AP-S Int. Symp., Newport Beach, CA, June 1995. 